die am einfachsten aus $(4,17)$ ableitbare Größe

$$
{ }^{(\prime \prime)} \overline{\Delta n^{2}}=v \varphi(2 \varphi-1) \text {; }
$$

welche erkennen läßt, in welcher Weise die zu verschiedenen $v$-Werten gehörigen Verteilungskurven der Ausgangsimpulse mit zunehmendem v zwar absolut immer breiter, im Relativmaßstab

$$
\sqrt{(a) \overline{\Delta n^{2}}} /{ }^{(a)} \bar{n}=\sqrt{(2 \varphi-1) / \nu \varphi}
$$

aber immer schmäler werden.

Zum Vergleich mit den Meßkurven z. B. von E. Fünfer, gewonnen mit Po- $\alpha$-Strahlen bei einem Multiplier mit vorgeschaltetem Leuchtschirm, müßte man erst von der differentiellen Kurve $(4,21)$ zur Integralkurve übergehen. Man erkennt, daß auch hier qualitative Úbereinstim- mung besteht. Doch zeigt auch hier der genaue Vergleich Abweichungen, indem die Fünferschen Meßkurven eine „Übergangsbreite“ besitzen, die mit zunehmendem ${ }^{(a)} \bar{n}$ immer kleiner zu werden scheint, während sie nach $(4,22)$ immer breiter werden müßte.

Mit diesem Beispiel mögen die Betrachtungen über die Anwendungen der statistischen Vervielfachertheorie vorerst abgeschlossen werden. Die Auswertung experimentell bestimmter Verteilungskurven für die Endimpulse auf Grund der Formel $(2,14)$ oder allenfalls der speziellen Beziehung $(4,2)$ muß so lange zurückgestellt werden, bis hinreichend genaue und reproduzierbare Meßkurven vorliegen, die mit einem Vervielfacher bestimmt wurden, dessen Verteilung des Verstärkungsfaktors ebenfalls durch genaue Messungen bekannt ist.

\title{
Leitfähigkeitsprofil der Sperrschicht des Kupferoxydul-Gleichrichters nach H-F-Messungen und seine Gliederung in Raumladungszonen*
}

\author{
Von Georg Pfotzer \\ Aus der Forschungsstelle für Physik der Stratosphäre, \\ Institut der Kaiser-Wilhelm-Gesellschaft in Weißenau \\ (Z. Naturforschg. 4 a, 691-703 [1949]; eingegangen am 22. Dezember 1948)
}

\begin{abstract}
Die Ergebnisse von Scheinwiderstandsmessungen am normalsperrenden $\mathrm{Cu}_{2} \mathrm{O}$-Gleichrichter werden in Abhängigkeit von der Frequenz bis $1 \mathrm{MHz}$ dargestellt und als Unterlagen für eine Analyse des Leitfähigkeitsprofils in der Sperrschichtzone diskutiert. Bereits aus dem Frequenzgang des Phasenwinkeltangens geht eine deutliche Gliederung der Randschicht in drei Bereiche mit verschiedener mittlerer Zeitkonstante hervor. Darüber hinaus läßt sich aber auf Grund eines hier entwickelten Näherungsverfahrens direkt das Profil der Wechselstromleitfähigkeit ableiten. Die unipolar leitende Zone breitet sich mit zunehmender Sperrspannung örtlich aus und schrumpft in Flußrichtung wieder zusammen. Spannungsunabhängige Begrenzungen dieses Gebietes infolge der besonderen chemischen Struktur der Randschicht treten nicht in Erscheinung. Damit wirg die Grundvorstellung der Schottkyschen Gleichrichtertheorie erneut in einprägsamer Weise bestätigt, daß die Unipolarität der Systeme Metall-Halbleiter durch stromgesteuerte Raumladungsverteilungen bedingt ist. Im Rahmen dieser Theorie kann der an das Mutterkupfer angrenzende Teil der Randschicht als Gebiet einer Donatorenerschöpfung (nach oben begrenzte Raumladungsdichte) gedeutet werden. Der anschlieBende Teil zeigt das Wechselstromverhalten eines Reservegebietes (steuerbare Raumladungsquellen), dessen Existenz bei Kupferoxydul damit erwiesen erscheint. Wider Erwarten endet die Reserveschicht nicht im quasineutralen Gebiet des Halbleiters, sondern in einem Raumladungsgebiet mit Erschöpfungscharakter. Nach einer neuen Hypothese von Schottky äußert sich hier ein Nebeneinander zweier Störstellenarten in der Randschicht.
\end{abstract}

Chottky und Deutschmann ${ }^{1}$ haben erstmalig Scheinwiderstandsmessungen an Kupferoxydul-Gleichrichtern mit Erfolg herangezogen,

1 W. Schottky u. W. Deutschmann, Physik. Z. 30, 839 [1929]. um Aufschluß über die Dicke der sogenannten Sperrschicht bei Systemen Metall-Halbleiter zu erlangen. Sie fanden, daß sich der Gleichrichter bei konstant gehaltener Gleich-Vorspannung einer

* Fußnote siehe Seite 692 . 
kleinen überlagerten Wechselspannung gegenüber im Tonfrequenzgebiet wie eine Ersatzschaltung nach Abb. 1 verhält.

Hierbei ist $R_{\mathrm{p}}$ ' ein von der Gleichspannungsbelastung stark abhängiger Widerstand, welcher im Sperrbereich etwa 100-mal größer ist als der nur geringfügig spannungsabhängige Vorwiderstand $R_{\mathrm{v}} . R_{\mathrm{p}}^{\prime}$ wurde als Widerstand der an das Mutterkupfer angrenzenden Randschicht gedeutet, in welcher der unipolare Leitungsmechanismus wirksam ist, $R_{\mathrm{v}}$ dagegen der restlichen, an die (sperrfreie) Gegenelektrode angrenzenden Kupferoxydulschicht zugeschrieben. Die Dicke der Sperrschicht $l$ wurde aus der Parallelkapazität $C_{\mathrm{p}}{ }^{\prime}$ nach Gl. (1) berechnet, worin $\varepsilon=12$ die Dielektrizitätskonstan-

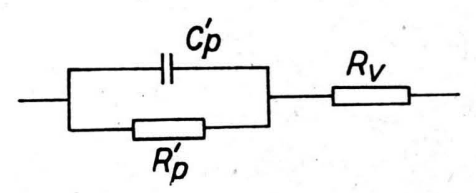

Abb. 1. Ersatzschaltung für den Kupferoxydul-Gleichrichter im Tonfrequenzgebiet nach Schottky und $\mathrm{Deutschmann}{ }^{1}$.

te des Kupferoxyduls und $q$ die Fläche des Gleichrichters beedeutet:

$$
l=\frac{\varepsilon q}{4 \pi C_{\mathrm{p}}^{\prime}} .
$$

Die nach Gl. (1) ermittelte Größenordnung von $l$ in Stärke von einigen 1000 Atomabständen und seine Zunahme mit wachsender Sperrspannung führten zu einer Kritik an der damals anerkannten ,gap“ (Spalt) oder Potentialberg-Vorstellung.** Danach sollte nämlich die Sperrschichtdicke unabhängig von der Spannung und nur etwa von der Größenordnung der Coulombschen Wirkungssphäre eines Atoms sein.

Im Gegensatz hierzu führte die moderne, von $\mathrm{S} \mathrm{ch} \mathrm{ot} \mathrm{t} \mathrm{k} \mathrm{y}{ }^{2}$ aufgestellte Raumladungstheorie der

* Die der Arbeit zugrunde liegenden Messungen wurden $1941 \mathrm{im} \mathrm{Z}$ entrallaboratorium der $\mathrm{S}$ i emens und H.alske A.G. ausgeführt und die Ergebnisse der Messungen in Form eines internen Aktenvermerkes niedergelegt. Die Analysenmethode wurde anschließend entwickelt, einige Schichtprofile wurden abgeleitet. Der Aktenvermerk sowie die handschriftlichen Aufzeichnungen zur Analyse gingen infolge der Kriegsereignisse verloren. Hrn. Prof. Sch ottky verdankt der Verf. schließlich die freundl. Uberlassung eines Auszuges aus dem Aktenvermerk mit den Meßergebnissen und die Möglichkeit, das Material erneut zu bearbeiten.
Randschichtgleichrichter zu einer widerspruchsfreien Deutung der Meßergebnisse, die von A. Schmidt 1941 auch für den Selengleichrichter grundsätzlich bestätigt wurden.

Ebenfalls im Jahre 1941 wurden nun vom Verf. im Rahmen einer technischen Aufgabe Scheinwiderstandsmessungen an Kupferoxydul-Gleichrichtern weit über das Tonfrequenzgebiet hinaus (bis $1 \mathrm{MHz}$ ) ausgeführt. Hierbei zeigte sich, daß das einfache Ersatzschaltbild nach Abb. 1 oberhalb des Tonfrequenzgebietes beim KupferoxydulGleichrichter nicht mehr ausreichend ist.

Dies gab den Anlaß zu einer eingehenden Bearbeitung der Messungen hinsichtlich ihres physikalischen Inhaltes. Die hierbei gewonnenen Ergebnisse führten schließlich zu neuen Erkenntnissen über das Leitfähigkeitsprofil des Kupferoxydul-Gleichrichters, welche aus den eingangs erwähnten Tonfrequenzmessungen noch nicht erschlossen werden konnten.

Im folgenden werden nach kurzem Eingehen auf meßtechnische Besonderheiten zunächst die Meßergebnisse mitgeteilt. Hierauf wird eine $\mathrm{Me}-$ thode zur Analyse des Leitfähigkeitsprofils entwickelt und zum Schluß das Leitfähigkeitsprofil des Kupferoxydul-Gleichrichters dargestellt und diskutiert.

\section{Messungen und Meßergebnisse}

\section{Definition des Scheinwiderstandes}

Der bekannte nichtlineare Zusammenhang zwischen Strom und Spannung bei Trockengleichrichtern gibt bei Wechselstrom-Messungen Anlaß zu Oberwellen. Es ist daher notwendig, eine genaue Definition der Meßgröße zu geben, wenn die Resultate mit anderen Messungen vergleichbar sein sollen.

Als Scheinwiderstand des Gleichrichters bezeichnen wir im folgenden denjenigen Wechselstromwiderstand bei konstanter Gleichspannungsbelastung $E_{\mathrm{g}}$, welcher als Quotient einer kleinen

** Über die historische Entwicklung und Klassifizierung der verschiedenen Grundvorstellungen sei vor allem auf die Arbeit W. S c h o t t k y, Zur Halbleitertheorie der Sperrschicht und Spitzengleichrichter, Z. Physik 113, 367 [1939], verwiesen.

2a W. Schottky, Z. Physik 113, 367 [1939], (Qualitative Darstellung). 2b W. Sch ot t ky u. E. S p e n k e, Wiss. Veröff. Siemens-Werke 18, 225 [1939], (Quantitative Darstellung). 2c W.Sch o t t ky Z. Physik 118, 539 [1941/42], (Vereinfachung und Erweiterung der Theorie).

3 A. S c h m id t, Z. Physik 117, 754 [1941]. 
überlagerten Wechselspannung $U_{0} \sin \omega t$ mit der Grundwelle des zugehörigen Wechselstromes $J_{0} \sin$ $(\omega t+\varphi)$ nach Betrag und Phase berechnet werden kainn.

Dieser Quotient geht bei sehr kleinen Frequenzen in den differentiellen Widerstand $R_{\sim}=d E_{\mathrm{g}} / d J_{\mathrm{g}}$ über. welcher aus der Gleichstromkennlinie $J_{\mathrm{g}}=f\left(E_{\mathrm{g}}\right)$ zu ermitteln ist.

\section{Meßtechnisches}

Der vorstehend definierte Scheinwiderstand wurde in der Brückenschaltung nach Abb. 2 gemessen, als geeignete Nachbildung wurde die Parallelschal- bietet die Messung bis etwa $-4 \mathrm{~V}$ keine besonderen Schwierigkeiten. Oberhalb dieser Spannung macht sich jedoch ein starkes Widerstandsrauschen störend bemerkbar, so daß die Wechselspannungs-Amplitude bis $\mathrm{zu} 50 \mathrm{mV}$ eff erhöht werden mußte, um den Rauschpegel genügend zu übertreffen. In diesem Spannungsgebiet ist aber die Nichtlinearität des Widerstandes so gering, daß die in I, 1 definierte Meßgröße nicht verfälscht wird. Besondere Vorsichtsmaßregeln erfordert die Messung des Scheinwiderstandes in Flubrichtung bei Gleichspannungen $>0,2 \mathrm{~V}$. Die hier ermittelten Zeitkonstanten des Gleichrichterwiderstandes im Bereich einiger $10^{-9}$ sec sind von der Größenordnung der Phasenkorrekturen auch sorgfältig bifilar gewikkelter Widerstandsnormalien. Auch Zuleitungen und

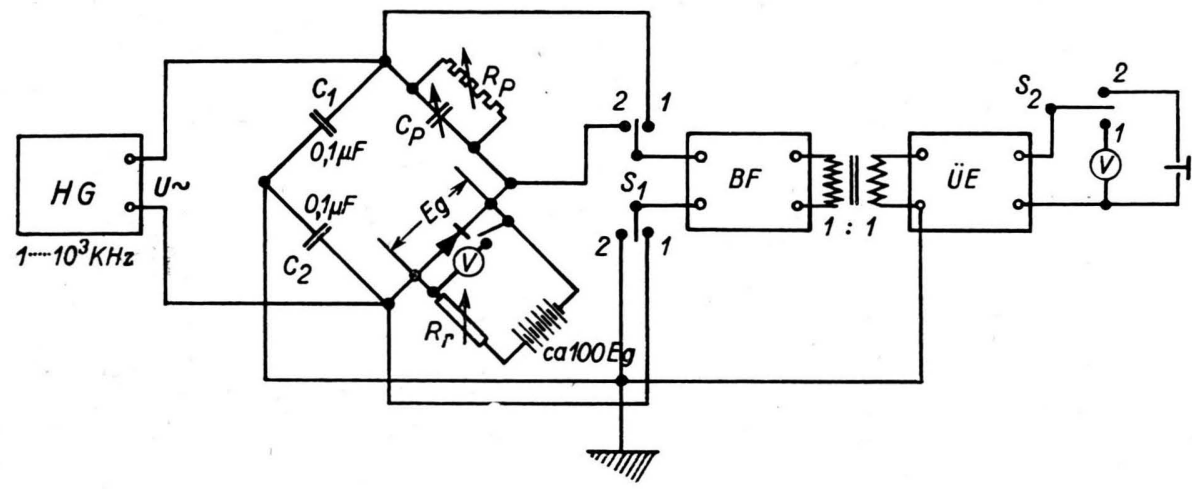

Abb. 2. Brückenschaltung zur Bestimmung des Scheinwiderstandes von Trockengleichrichtern in Abhängigkeit von der Gleich-Vorspannung und der Meßfrequenz. HG = Hochfrequenzgenerator, BF = Bandfilter, UE $=$ Thberlagerungsempfänger, $E_{\mathrm{g}}=$ Gleichvorspannung, $U \sim=$ Wechselspannung, $R_{\mathrm{r}}=$ Regelwiderstand, $C_{\mathrm{p}}$ = Kapazitätsnormal, $R_{\mathrm{p}}=$ Widerstandsnormal.

tung $R_{\mathrm{p}}-C_{\mathrm{p}}$ gewählt. Zur Verriegelung des Gleichstromkreises gegen den Wechselstromkreis und gleichzeitig als symmetrische Brückenwiderstände dienten die Kondensatoren $\mathrm{C}_{1}$ und $\mathrm{C}_{2}$. HG ist eiń Hochfrequenzgenerator mit festen Frequenzstufen 1:3 von $1 \mathrm{kHz}-1 \mathrm{MHz}$. Die Filterung der Diagonalspannung erfolgte mit Hilfe des Bandfilters BF, welches jeweils nur für die Grundwelle durchlässig war, ihre weitere Verstärkung mit Umsetzung der Meßfrequenz in den Hörbereich durch den Überlagerungsempfänger UE. Der Schalter $S_{1}$ diente zur Umschaltung auf Kontrollè der Brückenspannung über den ảs Röhrenvoltmeter geeichten Empfänger. Als Brückenwechselspannung wurden mit Ausnahme der Messungen bei -4 und $-6 \mathrm{~V}$ Vorbelastung $10 \mathrm{mV}_{\text {eff }}$ eingestellt, was einer Spannungsamplitude von $5 \mathrm{mV}$ eff am Gleichrichter entsprach. Die Gleichspannungsbelastung erfolgte durch Einprägung des Stromes über einen Regelwiderstand $R_{\mathrm{r}}$, welcher stets so groß gegen $R_{\mathrm{p}}$ gehalten wurde, daß er als Parallelwiderstand zum Gleichrichter nur eine Korrektur von etwa $1-2 \%$ erforderlich machte. Die Gleichspannung wurde vor und nach dem Brückenábgleich mit dem hochohmigen Voltmeter V kontrolliert, das Voltmeter während des Brückenabgleichs selbst jedoch abgeschaltet, um unnötige Korrekturen zu vermeiden. In Sperrichtung
Armatur des Gleichrichters machen sich bereits unangenehm bemerkbar. Der Armaturfehler wurde nun durch Fassung des Gleichrichters zwischen zwei kapazitätsarmen Blattfederkontakten klein gehalten, der Phasenfehler der Normalien durch Verwendung eigens für diesen $\mathrm{Z}_{\text {weck }}$ hergestellter Schichtwiderstände. Der Einfluß der Zuleitungen wurde durch eine Differenzmessung eliminiert: An Stelle des Gleichrichters wurde zunächst ein bestimmter induktionsfreier Schichtwiderstand (in diesem kritischen Bereich von der Größe 10-100 Ohm) eingesetzt und die Brücke mit den üblichen Normalien abgeglichen. Hierauf wurde der Schichtwiderstand durch den Gleichrichter ersetzt, die Einstellung des Widerstandsnormals unverändert gelassen und der Abgleich nunmehr durch Einstellung des Gleichrichterwiderstandes auf den Festwert durch Variation der Gleichspannung und durch Nachstellen der Kapazität vorgenommen. Differenzkapazität, Größe des Festwiderstandes und die nunmehr abgelesene Abgleichspannung $E_{\mathrm{g}}$ ergaben dann das zusammengehörende Wertetripel.

Ǔber die Meßgenauigkeit können den mir zur Verfügung stehenden Unterlagen leider keine präzisen Angaben mehr entnommen werden. Die Abgleichgenauigkeit in Sperrichtung läßt sich jedoch mit hinreichender Sicherheit auf etwa $\pm 0,5 \%$ beziffern. $\mathrm{Zu}$ - 
leitungen und Gleichspannungskreis mögen die Genauigkeit jedoch schätzungsweise auf etwa $\pm 2 \%$ in Sperrichtung herabgesetzt haben. Die Sicherheit der Phasenbestimmung in Flußrichtung war dagegen wesentlich geringer, etwa abnehmend von $\pm 5 \%$ bei $50 \mathrm{mV}$ auf etwa $\pm 30 \%$ bei Vorspannungen $>0,2 \mathrm{~V}$. Der Absolutwert des Scheinwiderstandes konnte jedoch auch im Flußgebiet auf etwa $\pm 2 \%$ genau bestimmt werden. Diese Angaben beruhen auf vorsichtigen Schätzungen auf Grund der Normaliengliederung und der Manipulationen bei der Messung.

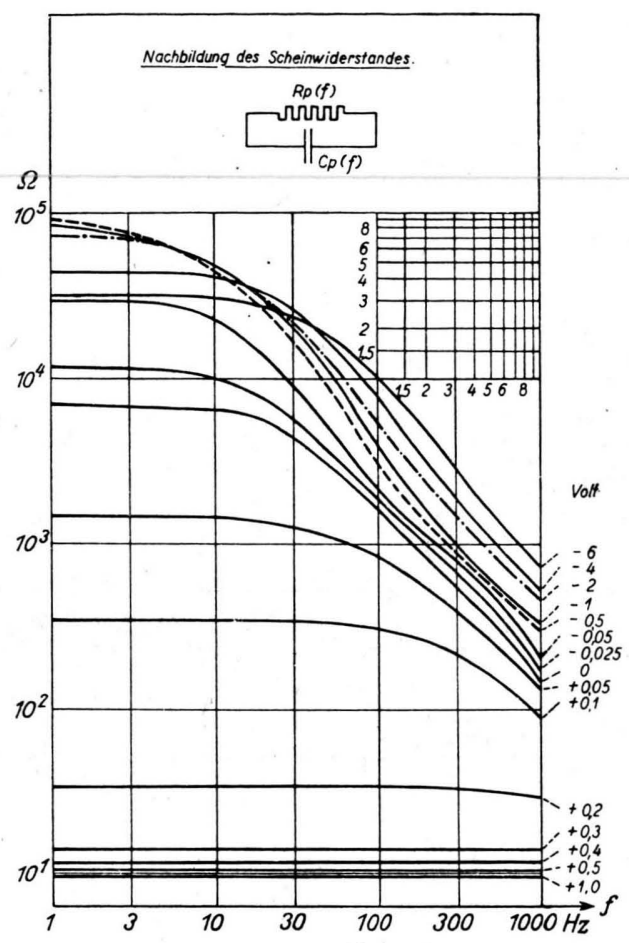

Abb. 3. Frequenzgang des Ohmschen Nachbildungswiderstandes $R_{\mathrm{p}}(f)$ eines Kupferoxydul-Gleichrichters (Siemens Rel gl 28), gemessen mît $U \sim=5 \mathrm{mV}$.

Scheibendurchmesser $7 \mathrm{~mm}$, Temperatur $20^{\circ} \mathrm{C}$.

\section{Darstellung der Meßergebnisse}

Die Meßergebnisse sind in den Abb. 3--6 dargestellt. Sie beziehen sich auf einen normalspe:-

* Die grundsätzliche Frequenzabhäng:gkeit des Scheinwiderstandes ist unabhängig von der Scheibengröße. Kapazitätswerte können praktisch flächenproportional umgerechnet werden, sofern man normal sperrende Gleichrichter unter sich vergleicht. Flächenproportionale Umrechnungen der Ohmschen Widerstände sind namentlich bei den kleinsten Typen ( 2 und $3 \mathrm{~mm} \varnothing$ ) weniger zutreffend, da hier systematische Unterschiede infolge fertigungstechnisch bedingte: Behandlungsvarianten möglich sind. Im Flußgebiet z. B. auch Beeinflussung der Widerstandsverhältnisse durch Änderung der Verhältnisse: Durchmesser der Gegenelektrode zu Scheibendurchmesser. renden Gleichrichter von Siemens und Halske der Type Rel gl 28a, Scheibengröße $7 \mathrm{~mm} \varnothing$, mit mittlerer Lage der Gleichstromkennlinie im Toleranzbereich*. Meßtemperatur $=20^{\circ} \mathrm{C}$. In Abb. 3 ist der unmittelbar gemessene Parallelwiderstand $R_{\mathrm{p}}$ (f) der Nachbildung in Abhängigkeit von der Frequenz fuir verschiedene Gleichspannungsbelastungen eingezeichnet. Seine Abnahme ist charakteristisch für den Schichtaufbau und zeigt, daß einzelne Teilwiderstände der Schicht mit zunehmender Frequenz offenbar kurzgeschlossen werden.

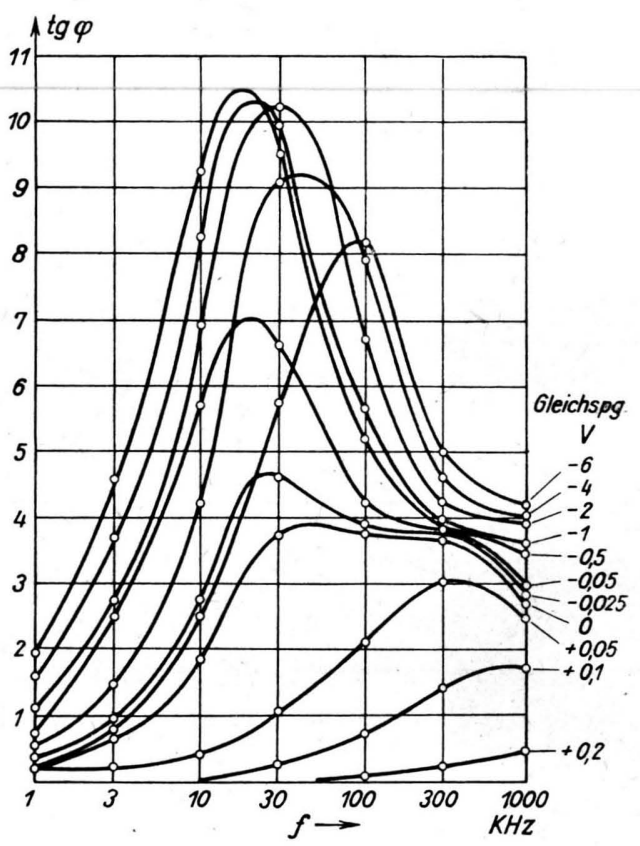

Abb. 4. Tangens des Phasenwinkels eines Kupferoxydul-Gleichrichters in Abhängigkeit von der Frequenz bei verschiedenen Gleichvorspannungen.

Abb. 4 enthält die Abhängigkeit des Phasenwinkeltangens von der Frequenz bei den verschiedenen Vorspannungen. Es ist:

$$
\operatorname{tg} \varphi=\omega R_{\mathrm{p}} C_{\mathrm{p}}
$$

$\left(R_{\mathrm{p}}\right.$ und $C_{\mathrm{p}}$ als Nachbildungselemente der Meßschaltung nach Abb. 2, $\omega=$ Kreisfrequenz).

Mit obiger Beziehung läßt sich aus Abb. 4 die Nachbildungskapazität entnolımen. Abb. 5 ergänzt die Darstellung $4 \mathrm{im}$ Flußgebiet und gibt gleichzeitig ein anschauliches Bild des Kapazitätsverlaufes bei konstanter Frequenz in Abhängigkeit von der Gleichspannung. Aus diesen Darstellun- 
gen 3-6 kann der Scheinwiderstand für jede Frequenz berechnet werden. Es ist:

$$
\Re(\omega)=\frac{R_{\mathrm{p}}}{1+\operatorname{tg}^{2} \varphi}-j R_{\mathrm{p}} \frac{\operatorname{tg} \varphi}{1+\operatorname{tg}^{2} \varphi} .
$$

Im Gegensatz zu den Abb. 3-5, welche nur einzelne Elemente des Scheinwiderstandes als Unterlagen für numerische Auswertungen enthalten, ist Abb. 6 als Übersichtsblatt für das Kennlinienfeld des Kupferoxydul-Gleichrichters gedacht. Neben den Absolutwerten des Scheinwiderstandes

$$
\left|\Re_{\mathrm{s}}\right|_{\omega}=\sqrt{\Re_{\mathrm{real}}^{2}+\mathfrak{R}_{\mathrm{im}}^{2}}=\frac{R_{\mathrm{p}}}{\sqrt{1+\operatorname{tg}^{2} \varphi}}
$$

mit den Zahlenwerten für $\operatorname{tg} \varphi$ ist auch der integrale Gleichstromwiderstand $E_{\mathrm{g}} / J_{\mathrm{g}}$ in Abhängigkeit von der Gleichspannung eingetragen. Die Abb. 6 ist vor allem für die technische Anwendung des Gleichrichters von Interesse, da sie unmittelbar die Beeinflussung des Güteverhältnisses Sperrwiderstand/Flußwiderstand durch kapazitive Überbrückung der Sperrschicht bei verschiedenen Frequenzen verdeutlicht.

Als Randbemerkung sei hier ein kurzer Hinweis auf eine Anwendungsmöglichkeit der Trockengleichrichter eingeflochten, welche zwar in reichem Maße in der Fernmeldetechnik ausgenützt wird, weiteren Kreisen im allgemeinen aber doch weniger geläufig ist. Es ist die Anwendung der Gleichrichter in vielseitigen Kombinationen als spannungsgesteuerte Wechselstromwiderstände. Die Abb. 5 und 6 stellen diese Eigenschaft des Gleichrichters heraus. Bei Frequenzen um $1 \mathrm{kHz}$ z. B. ändert sich der Absolutwert des Scheinwiderstandes um 4 Zehnerpotenzen, wenn die Steuerspannung im Bereich von $+0,5$ bis $-0,5 \mathrm{~V}$ variiert. Gleichzeitig mit der Änderung des Absolutwertes treten Phasendrehungen auf. Im Flußgebiet ist der Scheinwiderstand praktisch reell, in Sperrrichtung dagegen vorwiegend kapazitiv imaginär. Der Gleichrichter stellt somit in Sperrichtung praktisch eine (allerdings verlustreiche) Kapazität dar, welche durch eine Spannung steuerbar ist*.

\section{Diskussion der Meßergebnisse, Schichtenfolge}

Anknüpfend an die Arbeit von S chot tk y und Deutschmann ${ }^{1}$ haben wir in Abb. $7 a$ und $b$ als Beispiel für alle anderen Meßfolgen den Scheinwiderstand des Gleichrichters bei der Vorspan-

* Vgl. hierzu die kürzlich erschienenen Blätter im Arch. techn. Mess.: Fremdgesteuerte Meßgleichrichter von W. G e y g e r (ATM Z $52-7$, Z $52-8$, Oktober 1948).

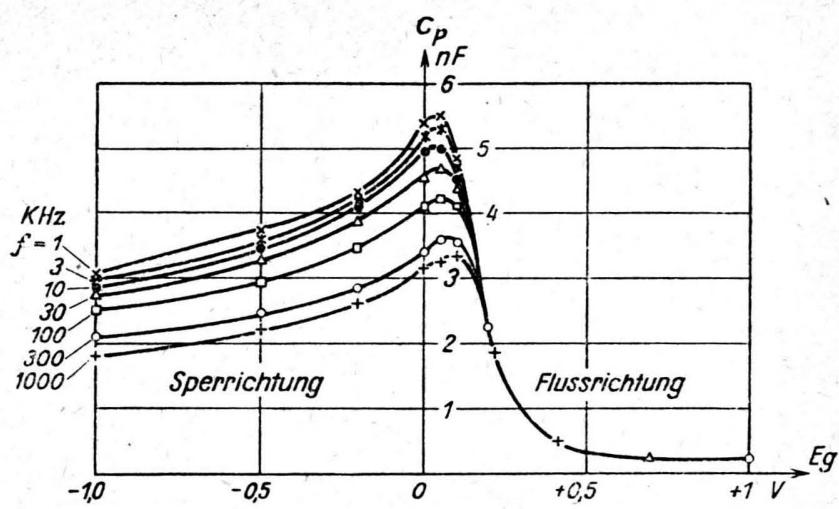

Abb. 5. Parallelkapazität eines Kupferoxydul-Gleichrichters $(7 \mathrm{~mm} \varnothing)$ in Abhängigkeit von der Gleichspannung bei verschiedenen Frequenzen.

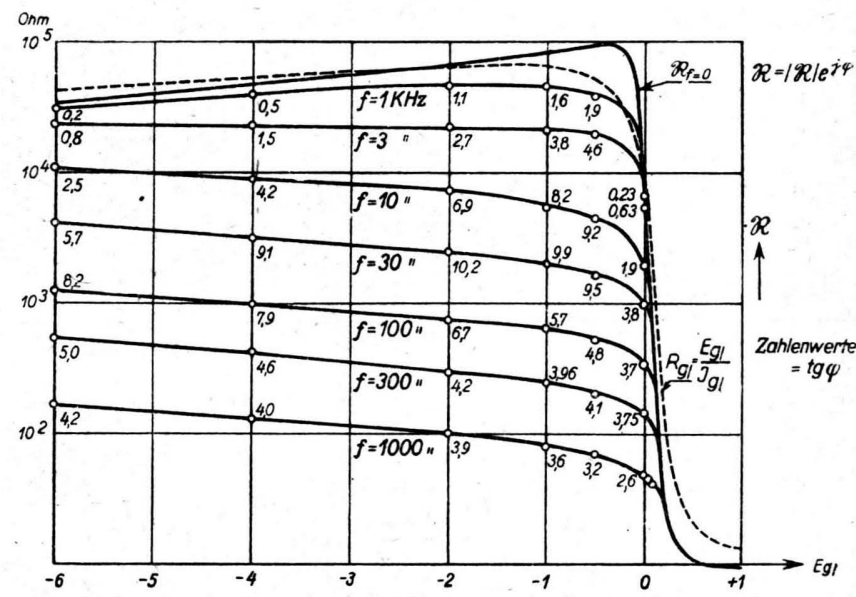

Abib. 6. Gleichstromkennlinie des Widerstandes eines $7 \mathrm{~mm}$ Kupferoxydul-Gleichrichters und Kennlinien des differentiellen Widerstandes in Abhängigkeit von derFrequenz.

nung 0 für verschiedene Frequenzen in die komplexe Ebene eingezeichnet. In 7 a sind die Koordinaten linear, in $7 \mathrm{~b}$ mit Rücksicht auf das Gebiet hoher Frequenzen logarithmisch geteilt (Parallele unter $135^{\circ}, \operatorname{tg} \varphi=$ konst.). Real- und Imaginärteil berechnen sich an Hand der Werte aus den Abb. 3 und 4 durch die Gln. (2) und (3).

Dem einfachen Ersatzschaltbild nach Abb.1, dessen Gültigkeit bei niederen Frequenzen Sch ottky und Deutschmann ${ }^{1}$ nachgewiesen haben, entspricht der durch die Punkte 0,1 und $3 \mathrm{kHz}$ gezogene Halbkreis (Abb. $7 \mathrm{a}$ ), welcher, in der logarithmischen Darstellung abgebildet, die Kurve $\mathfrak{g}+R_{\mathrm{v}}$ ergibt. (Die Basis des Halbkreises ist bekanntlich gleich dem Parallelwiderstand $R_{\mathrm{p}}{ }^{\prime}$ nach Abb. 1, und der Abschnitt auf der reellen Achse 
zwischen Ursprung und Halbkreis gleich $R_{r}$.) Aus den beiden Darstellungen, besonders evident aber aus Abb. 7b, geht nun hervor, daß das Ersatzschaltbild in diesem Falle bereits bei Frequenzen über $10 \mathrm{kHz}$ versagt; die Meßpunkte liegen weitab von der zugehörigen Ortskurve $\left(\mathfrak{S}+R_{\mathbf{v}}\right)$.

Trotzdem kann es aber, wie sich gleich zeigen wird, mit Vorteil zu einer rohen Klassifizierung einzelner Schichtabschnitte des Kupferoxyduls

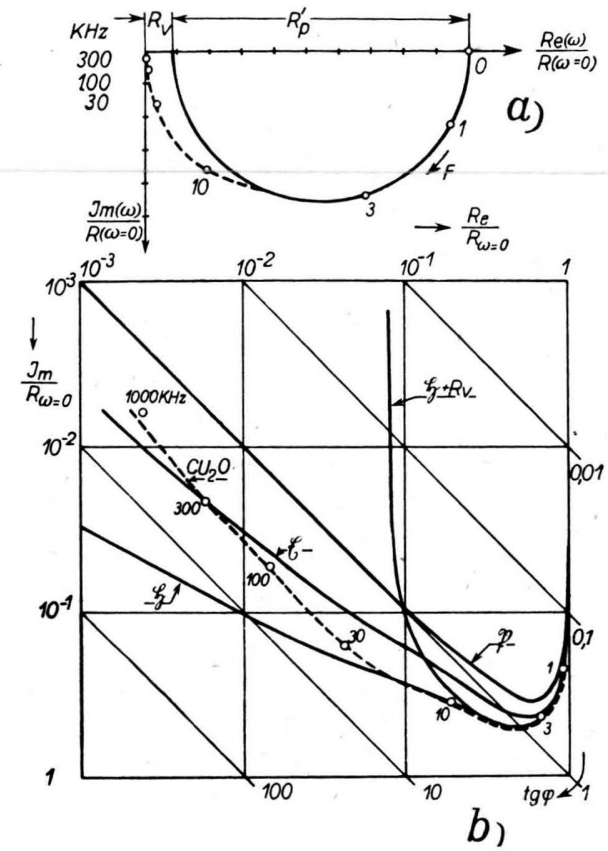

Abb. 7. Scheinwiderstandsdiagramm eines Kupferoxydul-Gleichrichters ohne Gleichspannungsbelastung (Meßpunkte) zum Vergleich mit Ortskurven des Scheinwiderstandes verschiedener Ersatzschaltungen. a) Linearer Maßstab, b) bilogarithmische Teilung. Erläuterungen im Text.

herangezogen werden. Insbesondere bietet uns die Frequenzabhängigkeit des Phasenwinkeltangens einer solchen Schaltung eine bequeme Möglichkeit hierzu. Diese läßt sich durch folgende Beziehung darstellen:

$$
\begin{array}{cc} 
& \operatorname{tg} \varphi=2 \operatorname{tg} \varphi_{\max } \frac{f / f_{\mathrm{m}}}{1+\left(f / f_{\mathrm{m}}\right)^{2}}, \\
\text { wo } & \operatorname{tg} \varphi_{\max }=\frac{R-R_{\mathrm{v}}}{2 \sqrt{R R_{\mathrm{v}}}} \\
\text { und } & f_{\mathrm{m}}=\frac{1}{2 \pi \tau_{\mathrm{p}}^{\prime}} \sqrt{\frac{R}{R_{\mathrm{v}}},}
\end{array}
$$

mit $R=R_{\mathrm{p}}^{\prime}+R_{\mathrm{v}} \equiv \Re_{(o=0}$

und der Zeitkonstante $\tau_{\mathrm{p}}^{\prime}=\omega R_{\mathrm{p}}^{\prime} C_{\mathrm{p}}^{\prime}$

ist. (Die Bezeichnungen entsprechen sinngemäß Abb. 1). Wie aus Gl. (4a) unmittelbar hervorgeht. durchläuft $\operatorname{tg} \varphi$ ein Maximum bei einer charakteristischen Frequenz $f=f_{\mathrm{m}}$. Trägt man nun $\operatorname{tg} \varphi$ in linearem Maßstab über dem Logarithmus der Frequenz auf, so erhält man eine für unsere Zwecke geeignete Darstellung in Form einer Glockenkurve mit der Symmetrieachse $f=f_{\mathrm{m}}$. In der Tat entsprechen die gemessenen $\operatorname{tg} \varphi$-Werte (Abb. 4) anfangs einem solchen Verlauf. Die Symmetrie verschwindet jedoch bei höheren Frequenzen in einer Weise, die auf Überlagerung einer zweiten, etwas niedrigeren Glockenkurve hinweist. In erster Näherung wird man also den $\operatorname{tg} \varphi$-Verlauf durch Superposition geeigneter Glockenkurven darstellen können.

Verfolgen wir die Kurvenform, bei $0,2 \mathrm{~V}$ beginnend, mit abnehmender Flußspannung über 0 bis zu hohen Sperrspannungen:

Der aufsteigende Ast bei $+0,2 \mathrm{~V}$ weist auf eine Schicht hin, die zwischen 100 und $1000 \mathrm{kHz}$ schwach durch. Verschiebungsströme überbrückt wird. Bei $+0,1 \mathrm{~V}$ zeigt sich bereits bei $1 \mathrm{MHz}$ die Andeutung eines Maximums, welches nach kleineren Frequenzen verschoben bei $50 \mathrm{mV}$ voll ausgeprägt ist. Nach Gl. (4a) manifestiert sich hierin eine Aufteilung der Schicht in zwei Bereiche mit verschiedener, mittlerer Zeitkonstante. Bei der Vorspannung $+50 \mathrm{mV}$ ist also eine neue Schicht mit größerer Zeitkonstante zusätzlich aufgebaut worden. Von $0 \mathrm{~V}$ an in Richtung wachsender Sperrspannung (-Zeichen) entwickelt sich schließlich das dominierende Tonfrequenzmaximum, welches einen extremen Schnitt im. Schichtaufbau anzeigt. Da die Unterlagerung des zweiten Maximums auch hier überall angedeutet ist, ergibt sich der zwingende Schluß, daß die anderen Schichten, zwar auch etwas verstärkt, aber grundsätzlich ihrer Struktur nach unverändert neben der Hauptschicht vorhanden sind. Diese beiden Schichten erscheinen im Tonfrequenzgebiet noch als Vorwiderstand nach der Ersatzschaltung (Abb.1). Dieser ist jedoch tatsächlich bei der charakteristischen Frequenz des Maximums $f_{\mathrm{m}}$ auch schon frequenzabhängig, was man leicht nachweist, wenn man $R_{\mathrm{v}}$ einmal aus den Daten des Maximums nach Gl. (4b) und gleichzeitig aus zwei aufeinanderfolgenden Werten des Schein- 
widerstandes bei den ersten beiden Meßfrequenzen bestimmt. Die Größe des Vorwiderstandes hat bereits bei $f_{\mathrm{m}}$ um etwa den Faktor 10 abgenommen. Eine Abtrennung der Einzelschichten kann also nur bei Berücksichtigung des ganzen Frequenzganges vorgenommen werden, worauf hier im Hinblick auf die folgende Behandlung der Probleme kurz hingewiesen sei.

Als summarisches Ergebnis haben wir hier die Existenz von mindestens 3 Schichtfolgen mit verschiedener mittlerer Zeitkonstante nachgewiesen. Diese werden mit abnehmender Sperrspannung und weiter in Richtung zunehmender Flußspannung nacheinander abgebaut.

\section{Entwicklung des Analysenverfahrens}

\section{Grundgleichung}

Wir haben uns nun die Aufgabe gestellt, durch eine eingehende Analyse der Frequenzabhängigkeit des Scheinwiderstandes zu detaillierteren Aussagen über den Aufbau der Randschicht zu gelangen. Ausgangspunkt unserer Überlegungen war folgende Grundidee:

Die verschiedenen Zeitkonstanten, welche wir aus den drei Teilabschnitten der $\operatorname{tg} \varphi$-Kurven herauslesen können, sind nur als Mittelwerte einer kontinuierlichen Verteilung aufzufassen. Falls es gelingt, diese Verteilung genauer $\mathrm{zu}$ bestimmen, läßt sich daraus auch die Verteilung der Leitfähigkeit ableiłen, wenn die Dielektrizitätskonstante (DK) durch das Auftreten von Störstellen nicht beeinflußt wird. Dies kann jedoch unbedenklich vorausgesetzt werden, da die DK durch die Resultate der Dipolmomente aller Atome bestimmt ist und Fehlordnungen der relativen Häufigkeit $10^{-6}$ praktisch nicht zur Geltung kommen dürften.

Wir denken uns nun die Kupferoxydulschicht parallel zu den Metallelektroden in einzelne Scheiben derart zerlegt, daß die Leitfähigkeit innerhalb einer Teilschicht in einem Bèreich zwischen $\varkappa$ und $\varkappa+\Delta \varkappa$ abgegrenzt wird. Die Dicke $\Delta x$ einer solchen Schicht ist dann ein Maß für den Einfluß des Bereiches mit der Leitfähigkeit $\varkappa$ auf den Gesamtwiderstand und kann bei geeigneter Wahl der Maßeinheit zu einer Verteilungsfunktion $f(x)$ durch

$$
\Delta x=f(\varkappa) \Delta \varkappa
$$

in Beziehung gesetzt werden.

Der Schichtdicke $\Delta x$ ist nun eine Teilkapazität

$$
\Delta C=\frac{\varepsilon}{4 \pi \Delta x}=\frac{\varepsilon}{4 \pi f(\varkappa) \Delta \varkappa}
$$

und ein Ohmscher Teilwiderstand

$$
\Delta R=\frac{\Delta x}{\varkappa}=\frac{f(\varkappa) \Delta x}{\varkappa}
$$

zuzuordnen (alle Werte beziehen sich auf die Flächeneinheit). Die Kapazität ist dem Widerstand parallel geschaltet, so daß als komplexer Leitwert

$$
\mathfrak{g}(\varkappa, \omega)=\frac{x}{f(x) \Delta x}+j \frac{\omega \varepsilon}{4 \pi f(x) \Delta x}
$$

resultiert und als Scheinwiderstand:

$$
\Delta \Re(\omega, \varkappa)=\frac{1}{\varkappa+j \omega \varepsilon / 4 \pi} f(\varkappa) \Delta \varkappa .
$$

Der Scheinwiderstand der ganzen Oxydulschicht ergibt sich dann als Summe der Widerstände aller

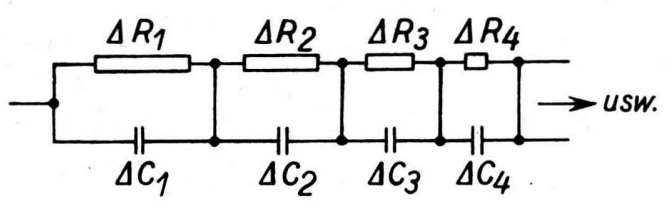

Abh. 8. Ersatzschaltbild für Schichten mit ortsabhängiger Leitfähigkeit.

Teilschichten. Er entspricht der Ersatzschaltung durch einen Kettenleiter nach Abb. 8.

Bei genügend feiner Unterteilung der Schicht können wir schließlich auch den Grenzübergang $\Delta x \rightarrow 0$ vollziehen 'und an Stelle der Summe sofort das Integral der Gl. (6a) anschreiben. Es ist:

$$
\Re[\omega, f(x)]=\int_{\varkappa_{1}}^{\varkappa_{2}} \frac{1}{\varkappa+j \omega \varepsilon / 4 \pi} f(x) d x .
$$

Liegt nun eine gemessene Frequenzkurve $\Re(\omega)$ vor, so läßt sich $f(\varkappa)$ durch Auflösung der Integralgleichung (6a) unter gewissen Voraussetzungen bestimmen, auf die wir nun etwas näher eingehen müssen.

Denken wir uns zunächst den Scheinwiderstand einer Schichtfolge mit örtlich variierendem $x$ bei so kleinen Frequenzen gemessen, daß der Verschiebungsstrom gegenüber dem Leitungsstrom in allen Schichten noch nicht ins Gewicht fällt, dann erlaubt diese Messung lediglich die Bestimmung eines Mittelwertes von $x$ über die ganze Schicht. 
Steigert man die Frequenz, so wird der Verschiebungsstrom bei den kleinsten $\varkappa$-Werten der Schicht allmählich merklich, der Scheiṇwiderstand beginnt in solcher Weise abzunehmen, daß eine Unterteilung der Schicht in Bereiche mit kapazitiver Überbrückung und frequenzunabhängige Bereiche möglich ist. Diese Klassifizierung entspricht bereits dem Ersatzschaltbild nach Abb. 1, wonach man ein mittleres $\varkappa$ für den Bereich mit der $\mathrm{Ka}$ pazitätslänge $l$ und einen hiervon abweichenden Mittelwert für den Restteil der Schicht angeben kann. Bei noch weiterer Steigerung der Frequenz werden schließlich einige Schichten total kurz geschlossen. Die Abnahme des Parallelwiderstandes der Nachbildung nach Abb. 3 mit der Frequenz gibt, worauf wir in Abschnitt I, 3 schon kurz hingewiesen haben, ein anschauliches Bild dieser sukzessiven totalen Kurzschlüsse. Die Ausbreitung der erst unvollkommenen kapazitiven Überbrükkung wirkt sich dagegen in der Abnahme der Nachbildungskapazität aus (Abb.5; zunehmender Abstand der Kondensatorbelegungen).

Man hat somit bei jeder Frequenz drei Gebiete der Häufigkeitsverteilung von $\varkappa$ zu unterscheiden:

1. Ein Gebiet, das wegen kapazitiver Kurzschlüsse überhaupt keinen Beitrag mehr zur reellen Komponente des Scheinwiderstandes gibt,

2. ein Leitfähigkeitsintervall, in welchem der Verschiebungsstrom etwa gleich groß ist wie der Leitungsstrom, und

3. Schichtanteile, in welchen der Leitungsstrom überwiegt, deren Scheinwiderstand also noch frequenzunabhängig ist.

Die Aufgabe der Transformation des $\omega-\Re(\omega)$ Profils in ein $x-f(x)$-Profil besteht nun in der Abgrenzung solcher Leitfähigkeitsintervalle für jede Frequenz.

Die exakte Lösung der Integralgleichung (6a) erschöpft automatisch die Möglichkeiten differentieller Aussagen über die vorliegende $x$-Verteilung innerhalb des Frequenzintervalls, in welchem der Gang des Scheinwiderstandes ermittelt wurde. Die Aussage über das Gewicht eines Bereiches mit bestimmtem $\varkappa$ ist um so genauer, je vollständiger der Kurzschluß für die vorgelagerten Schichten bereits vollzogen ist. Das Verhältnis des Verschiebungsstromes $U_{\sim} \omega \varepsilon / 4 \pi \Delta x \mathrm{zum}$ Leitungsstrom $U_{\sim} \varkappa / \Delta x$ erscheint somit als Maß für die Zuverlässigkeit der Lösung. Für eine noch so rohe Aussage wird man mindestens

$$
\omega \varepsilon / 4 \pi \varkappa \approx 1
$$

fordern müssen. Das bedeutet aber, daß wir mit $\varepsilon=12$ für $\mathrm{Cu}_{2} \mathrm{O}$ und $\omega=2 \pi \cdot 10^{6} \mathrm{sec}^{-1}$, entsprechend unserer größten Meßfrequenz, ein einigermaßen zutreffendes Bild der Leitfähigkeitsverteilung nur bis $\varkappa=6 \cdot 10^{-6} \mathrm{Ohm}^{-1} \mathrm{~cm}^{-1}$ erwarten dürfen. Der Restwiderstand jenseits dieses Grenzwertes erscheint bei der Frequenzanalyse als ungegliederter, frequenzunabhängiger Vorwiderstand, unabhängig von der oberen Begrenzung des Integrals. Für die Rechnung kann daher ohne weiteres auch $\varkappa=\infty$ zugelassen werden, sofern nur das Integral mit der Verteilungsfunktion $f(x)$. welche dem Frequenzgang bis $1 \mathrm{MHz}$ entspricht. für $\varkappa=\infty$ konvergiert. Der Vorwiderstand kann so in das Integral mit einbezogen werden, was für die numerische Auswertung bequem ist. Wir haben uns nur beim Endresultat daran zu erinnern. daß der Extrapolation $f(x)$ über die Frequenzschranke hinaus keine physikalische Realität zu entsprechen braucht.

Was wir nun weiter anstreben, ist eine örtliche Zuordnung der $x$-Werte. Eine solche ist aber nur dann möglich, wenn $\varkappa(x)$ eine eindeutige Funktion der Entfernung vom Rand ist. Alle Erfahrungen sprechen nun dafür, daß die Leitfähigkeit innerhalb der Kupferoxydulschicht von hohen Werten an der Gegenelektrode monoton auf die niederen Werte an der Mutterkupferseite abfällt. Wir können daher die Gl. (6a) in.die Form

$$
\Re(\omega)=\int_{x_{1}}^{x_{2}} \frac{1}{x(x)+j \omega \varepsilon / 4 \pi} d x
$$

umschreiben, da unter obiger Voraussetzung auch

$$
\lim _{\Delta x \rightarrow 0} \frac{\Delta x}{\Delta x}=\frac{d x}{d x} \equiv f(x)
$$

erlaubt ist.

\section{Näherungsweise Lösung der Grundgleichung}

a) Vorbemerkungen. Die Lösung der Integralgleichung (6b) läßt sich nun nicht in geschlossener Form darstellen, weil $\mathfrak{R}(\omega)$ nur als empirisch bestimmter Verlauf vorliegt. Wir werden daher den wahren $x$ - $x$-Verlauf stuickweise durch geeignete Näherungen darstellen, deren 
Scheinwiderstand durch Integration der Gl. (6a) bzw. (6 b) berechnet werden kann. Durch Vergleich mit dem gemessenen Frequenzgang lassen sich dann die erforderlichen Konstanten ermitteln. Für die Auswahl der Näherungsfunktion gibt es außer bestimmten physikalischen Fragestellungen und der ökonomie der numerischen Auswertung keine besonderen Einschränkungen.

Physikalisch waren für uns neben der $A b$ trennung homogener Bereiche (Bahnwiderstand. Erschöpfungsrandschicht, worauf wir w. u. ausführlich eingehen) besonders Schichten mit exponentiellem bzw. linearem Gefälle der. Leitfähigkeit von Interesse. Ein exponentielles, örtlich festliegendes Gefälle wurde nämlich von F. W a i bel ta an verhältnismäßig dicken Kupferoxydulschichten mit schwacher Unipolarität durch schrittweises Abätzen von Teilschichten nachgewiesen. Es war daher naheliegend, beim technischen Gleichrichter mit großer Richtwirkung einem solchen, entsprechend steileren, auf enge Bereiche konzentrierten Gefälle nachzuspüren. Ein lineares Gefälle $\left(x \sim x^{2}\right)$ dagegen ist nach der Schottkyschen Theorie für eine besondere Art von Raumladungszonen charakteristisch. Unmittelbare physikalische Beweiskraft kommt speziellen Näherungsfunktionen allerdings nur dann zı, wenn die Abhängigkeit des Scheinwiderstandes von der Frequenz durch eine einzige Funktion beschrieben werden kann. Der folgende Vergleich der Frequenzabhängigkeit der Scheinwiderstände einer ,homogenen“, einer ,parabolischen“ und einer ,exponentiellen“ Schicht mit einem gemessenen Verlauf wird uns allerdings zeigen, daß das Schichtprofil keinem dieser einfachen Typenfälle für sich allein entspricht, sondern zusammengesetzter Natur sein muß.

Bevor wir nun den Frequenzgang der einzelnen Näherungswiderstände angeben, sei noch darauf verwiesen, daß wir für die Exponential- und die Parabelschicht zunächst beliebig hohe Leitfähigkeiten zulassen wollen. Die Integrale konvergieren für $\varkappa_{2} \rightarrow \infty$ und führen so auf den charakteristischen Frequenzgang der betr. Elementarfunktion, sobald mit steigender Frequenz $\omega \varepsilon / 4 \pi \varkappa_{1}>1$ geworden ist. Widerstände zweiseitig begrenzter Bereiche sind darstellbar durch die Differenz zweier Elementarfunktionen mit Parametern (Eigenwerten), welche von der Begrenzung des

4a F. W a i b e l, Wiss. Veröff. Siemens-Werke 15, 75 [1936]; 4b Z. techn. Physik 11, 366 [1935].
Bereiches abhängen. Also:

$$
\Re(\omega)=\int_{\varkappa_{1}}^{\varkappa_{2}}=\int_{\varkappa_{1}}^{\infty}-\int_{\varkappa_{2}}^{\infty} .
$$

Ersichtlich nähert sich $\Re(\omega)$ mehr und mehr dem Frequenzgang einer zweiseitig begrenzten homogenen Schicht, je flacher das Leitfähigkeitsgefälle ist und je näher die Grenzen zusammenrücken. Der charakteristische Frequenzgang eines bestimmten Gefälles wird somit immer durch die stets vorhandene Begrenzung mehr oder weniger gestört. Dies ist auch der Grund, weshalb sich nur extrem starke Gefälle der Leitfähigkeit durch HF-Messungen erschließen lassen.

b) Elementarfunktionen. Homogene Schicht. Der Grenzfall konstanter Leitfähigkeit innerhalb einer Schicht der Dicke $d$ entspricht der Parallelschaltung eines Ohmschen Widerstandes $R=d / \varkappa$ mit einer Kapazität $C=\varepsilon / 4 \pi d$, wenn wir alle Größen auf die Flächeneinheit beziehen. Wir haben hier nur Gl. (3) in geeigneter Weise umzuformen und vermerken, daß nunmehr $R_{\mathrm{p}}$ und $C_{\mathrm{p}}$ als frequenzunabhängige Konstante $\mathrm{zu}$ behandeln sind. Mit $R_{\mathrm{p}} C_{\mathrm{p}}=\tau=\varepsilon / 4 \pi \varkappa$ und $\omega \tau=\Omega$ erhält man die unserem weiteren Vorgehen angepaßte Form:

$$
\Re_{\mathrm{H}}(\Omega)=\frac{d}{\varkappa}\left(\frac{1}{1+\Omega^{2}}-j \frac{\Omega}{1+\Omega^{2}}\right) .
$$

Hierbei bedeuten $\tau$ die Zeitkonstante der Schicht, $\varepsilon$ die DK des Materials, $\omega$ die Kreisfrequenz und $\Omega$ ein dimensionsloses, in Einheiten der reziproken Zeitkonstante ausgedrücktes Frequenzmaß, welches in diesem speziellen Falle mit dem $\operatorname{tg} \varphi$ identisch ist. Bezeichnen wir den Klammerausdruck mit $\mathfrak{F}_{\mathscr{\gamma}}$, so ist dieser Ausdruck als Elementarfunktion des Scheinwiderstandes einer homogenen Schicht mit dem Eigenwert $\tau=\varepsilon / 4 \pi \varkappa$ aufzufassen, welche, mit einem Gewichtsfaktor $d / \varkappa$ multipliziert, den Gesamtwiderstand ergibt.

Exponentialschicht. Die Elementarfunktion einer Schicht mit exponentieller Zunahme der Leitfähigkeit nach Gl. (9)

$$
\varkappa(x)=\varkappa_{\mathrm{a}} e^{a x}
$$

berechnen wir durch Integration der Grundgleichung in der Form (6a). Man hat dann nur mit Berücksichtigung von (9) und (5b) 


$$
f(\varkappa) \equiv \frac{d x}{d \varkappa}=\frac{1}{\alpha \varkappa}
$$

und als Grenzen des Integrals $x=\varkappa_{\mathrm{a}}$ bzw. $x=\infty$ einzusetzen und erhält unter Weglassung der Zwischenrechnung

$\Re_{\mathrm{e}}\left(\Omega_{\mathrm{a}}\right)=\frac{1}{a \varkappa_{\mathrm{a}}}\left\{\frac{\operatorname{arctg} \Omega_{\mathrm{a}}}{\Omega_{\mathrm{a}}}-j \frac{\ln \left(1+\Omega_{\mathrm{a}}^{2}\right)}{2 \Omega_{\mathrm{a}}}\right\}$.

$\Omega_{\mathrm{a}}$ ist wieder auf Einheiten einer reziproken Zeitkonstante bezogen, hier aber derjenigen, welche der Anfangsleitfähigkeit $\varkappa_{\mathrm{a}}$ der Schicht entspricht. Bezeichnen wir analog unserem Vorgehen im vorigen Abschnitt den Klammerausdruck als Elementarfunktion der Exponentialschicht mit $\mathfrak{F}_{\gamma_{\mathrm{a}}}$, so ist ihr Eigenwert durch die Anfangsleitfähigkeit der Halbraumschicht mit $\tau_{\mathrm{a}}=\frac{\varepsilon}{4 \pi \kappa_{\mathrm{a}}}$ und der Gewichtsfaktor durch den Exponenten $\alpha$ und $\varkappa_{\mathrm{a}}$ festgelegt mit $1 / \alpha \varkappa_{\mathrm{a}}$ als Gesamtwiderstand der Schicht bei $\omega=0$.

Parabelschicht. In gleicher Weise ergibt sich die Elementarfunktion $\mathfrak{P}$ für die Schmiegungsparabel indem man

$$
\varkappa(x)=\varkappa_{\mathrm{a}}\left(\frac{x+b}{b}\right)^{2}
$$

setzt.

Die Normierung ist so getroffen, daß für $x=0$; $\varkappa=\varkappa_{\mathrm{a}}$, also gleich dem Anfangswert des Schichtabschnittes ist. Die Integration ergibt für den Halbraum $x=x_{\mathrm{a}}$ bis $x=\infty$

$$
\begin{gathered}
\Re_{\mathrm{p}}\left(\Omega_{\mathrm{a}}\right)=\frac{b}{x_{\mathrm{a}}} \frac{1}{2 \sqrt{2 \Omega_{\mathrm{a}}}}\left\{w_{1}+w_{2}-j\left(w_{1}-w_{2}\right)\right\}, \\
w_{1}=\operatorname{arctg} \sqrt{\frac{2 \Omega_{\mathrm{a}}}{1-\Omega_{\mathrm{a}}},} \\
w_{2}=\frac{1}{2} \ln \frac{1+\left(\sqrt{2 \Omega_{\mathrm{a}}}+1\right)^{2}}{1+\left(\sqrt{2 \Omega_{\mathrm{a}}}-1\right)^{2}} .
\end{gathered}
$$

Die Elementarfunktion der Parabelschicht ist somit

$$
\mathfrak{P}_{\varkappa_{\mathrm{a}}}=\Re_{\mathrm{p}}\left(\Omega_{\mathrm{a}}\right) \frac{\varkappa_{\mathrm{a}}}{b},
$$

mit gleicher Bedeutung der Eigenwerte wie bei der Exponentialschicht.

c) Vergleich der Frequenzabhän- gigkeit des Scheinwiderstandes einer $\mathfrak{b}$, $\mathfrak{x}$ und $\mathfrak{P}$-Schicht mit einem gemessenen Scheinwiderstand bei $E_{\mathrm{g}}=0$.

Die charakteristischen Unterschiede der drei Elementarfunktionen ergeben sich am deutlichsten aus dem Gang des Phasenwinkels mit der Frequenz. Bei kleinen Frequenzen steigt der $\operatorname{tg} \varphi$ zunächst bei allen Funktionen mit $\Omega$ an. Dieser Gang bleibt jedoch nur bei der $\mathfrak{S}$-Schicht für alle Frequenzen erhalten. Bei der $\leftleftarrows$-Schicht dagegen

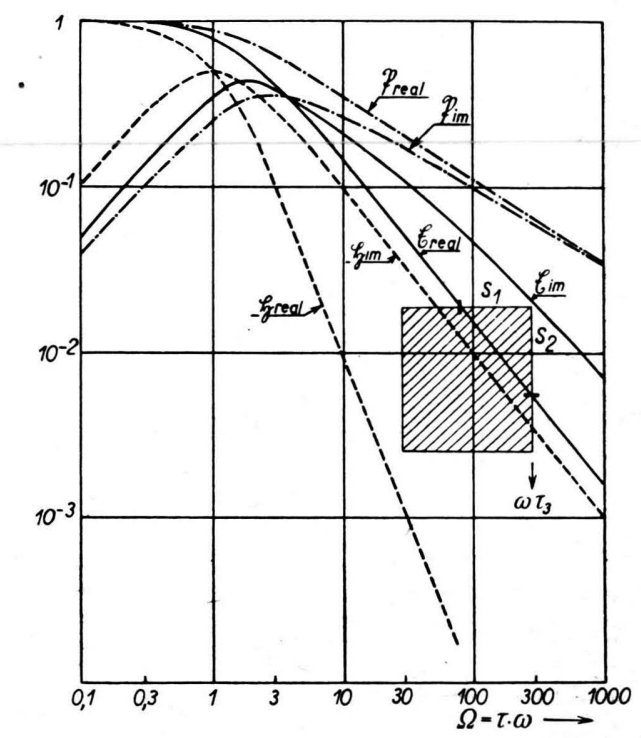

Abb. 9. Reelle und imaginäre Anteile der Elementarfunktionen des Scheinwiderstandes einer Schicht mit der Leitfähigkeit $\varkappa=$ konst. (homogene Schicht) $\mathfrak{L}$, bzw. $\varkappa \sim(x+b)^{2}$ (Parabelschicht) P, bzw. $x \sim e a x$ (Exponentialschicht) $\leftleftarrows$. Weitere Erläuterungen im Text.

wird mit $\Omega \gg 1$ ein asymptotischer Gang mit $\ln \Omega$ erreicht, bei der $\mathfrak{B}$-Schicht schließlich ein so langsames Anwachsen, daß $\operatorname{tg} \varphi=1$ angestrebt wird. Die Abnahme der reellen Teile als Maß für den sukzessiven Kurzschluß der Teilschichten mit der Frequenz erfolgt bei der $\mathfrak{S}$-Schicht für $\Omega \gg 1$ mit $1 / \Omega^{2}$, bei der $\check{5}$-Schicht mit $1 / \Omega$ und schließlich bei der $\mathfrak{F}$-Schicht mit $1 / \sqrt{\Omega}$. Diese Verhältnisse sind in Abb. 9 dargestellt. Je nach der vorliegenden Verteilung von $x$ wird man den Gang des Scheinwiderstandes stückweise mehr mit der einen oder anderen Funktion beschreiben können. Als Beispiel für einen typischen Verlauf des gemessenen Scheinwiderstandes zwischen diesen Elementarfunktionen können wir nochmals Abb. 7b heranziehen. Hier sind außer 
den Meßpunkten* für den Scheinwiderstand bei $E_{\mathrm{g}}=0$ auch die Ortskurven der drei Elementarfunktionen eingezeichnet. Der Vergleich zeigt, daß die Meßwerte zu keiner Ortskurve genau. besser aber zu der $\mathfrak{H}$ - und $\mathfrak{E}$-, als zur $\mathfrak{B}$-Funktion passen, und daß wir offensichtlich mit einer gröberen Intervallteilung bei Bevorzugung der ersteren auskommen werden.

Mit dieser Festlegung auf die $\mathfrak{h}$ - und $\mathfrak{E}$-Schicht reduziert sich die tatsächliche Arbeit bei der Auswertung auf die Aufgabe, die Einteilung der Schicht in Leitfähigkeitsintervalle dem vorliegenden Frequenzgang möglichst zwanglos anzupassen.

\section{Bestimmung der Konstanten}

Wir haben nun bereits in Abschn. II eine rohe Unterteilung der Schicht in drei frequenzabhängige Bereiche vornehmen können. Die anfängliche Abnahme des Scheinwiderstandes mit der Frequenz entspricht ganz klar einer homogenen Schicht, deren Einfluß bei kleinen Frequenzen weitaus überwiegt. Die beiden folgenden $\mathrm{Ab}$ schnitte, bei kleinen Frequenzen durch den vorerst frequenzunabhängigen Vorwiderstand $R_{\mathrm{v}}$ nach Abb. 1 repräsentiert, werden wir als Teile von Exponentialschichten auffassen und ihren Scheinwiderstand durch zwei Elementarfunktionen $\tilde{r}_{1,2}$ und $\mathfrak{r}_{3}$ darstellen, deren Exponenten $\alpha_{1,3}$ und $\alpha_{3}$ zu bestimmen sind. Die Mittelschicht erstreckt sich vom Leitwert $\varkappa_{1,2}$ der homogenen Schicht bis zum Leitwert $\varkappa_{3}$, bei welchem die dritte Schicht beginnt. Wir werden daher von der Elementarfunktion $\mathfrak{F}_{1,2}$ denjenigen Teil abzuziehen haben, welcher jenseits $\varkappa_{3}$ liegt, das ist aber die Elementarfunktion $\mathfrak{F}_{3}$ mit dem Exponenten $a_{1,3}$. Auf Grund dieser. Überlegungen gelangen wir nun $\mathrm{zu}$ folgendem Gleichungssystem für die Schichtkonstanten. Der Scheinwiderstand bei einer bestimmten Frequenz $f_{i}$ ist die Summe der Scheinwiderstände aller Teilschichten. Diesẽ ist daher dem tatsächlich gemessenen Wert $\mathfrak{R}\left(f_{\mathrm{i}}\right)$ gleichzusetzen.

$$
\begin{aligned}
\Re\left(f_{\mathrm{i}}\right)=\frac{d_{1}}{\varkappa_{1,2}} \mathfrak{S}_{1}\left(f_{\mathrm{i}}\right) & +\frac{1}{a_{1,3} \varkappa_{1,2}} \mathfrak{}_{1,2}\left(f_{\mathrm{i}}\right) \\
& -\frac{1}{a_{1,3} \varkappa_{3}} \mathfrak{F}_{3}\left(f_{\mathrm{i}}\right)+\frac{1}{\alpha_{3} \varkappa_{3}} \mathfrak{F}_{3}\left(f_{\mathrm{i}}\right),
\end{aligned}
$$

\footnotetext{
* Die Bedeutung der gestrichelten Kurve in Abb. $7 \mathrm{~b}$ s. Abschn. IV.
}

oder mit Zusammenfassung der Elementarfunktionen und der Konstanten:

$$
\Re\left(f_{\mathrm{i}}\right)=A \mathfrak{S}_{1}\left(f_{\mathrm{i}}\right)+B \mathfrak{E}_{1,2}\left(f_{\mathrm{i}}\right)+C \mathfrak{E}_{3}\left(f_{\mathrm{i}}\right),
$$

wo

ist.

$$
\begin{gathered}
A=d_{1} / \varkappa_{1,2}, \quad B=1 / \alpha_{1,3} \varkappa_{1,2}, \\
C=1 / \varkappa_{3}\left(1 / \alpha_{3}-1 / \alpha_{1,3}\right)
\end{gathered}
$$

Die Werte der Elementarfunktionen für eine beliebige Frequenz lassen sich nun aus der Darstellung in Abb. 9 entnehmen, wenn die Zeitkonstanten bekannt sind. Zu deren Ermittlung machen wir von der aus Abb.4 hervorgehenden Tatsache Gebrauch, daß der Einfluß der homogenen Schicht bei kleinen Frequenzen überwiegend ist. Die Zeitkonstante der homogenen Schicht läßt sich danach in einfacher Weise aus dem $\operatorname{tg} \varphi$ bei kleinen Frequenzen bestimmen. Es ist:

$$
\tau_{1,2}=\operatorname{tg} \varphi / \omega=\varepsilon / 4 \pi \varkappa_{1,2} .
$$

Damit sind die Abszissenwerte $\Omega_{\mathrm{i}}$ für $\mathfrak{\mathfrak { g }}_{1}$ und $\tilde{\mathfrak{E}}_{1,2}$ in Abb. 9 durch

$$
\Omega_{\mathrm{i}}=\tau_{1,2} \omega_{\mathrm{i}}=\tau_{1,2} 2 \pi f_{\mathrm{i}}
$$

für jede Frequenz $f_{i}$ festgelegt. Ebenso die Leitfähigkeit $\varkappa_{1,2}$ der homogenen Schicht, welche aus Gründen der Stetigkeit mit der Anfangsleitfähigkeit der Mittelschicht identisch sein muß. Die Zeitkonstante der dritten Schicht ermitteln wir aus dem Verhältnis der reellen Komponente des gemessenen Scheinwiderstandes bei den beiden höchsten Meßfrequenzen $0,3 \mathrm{MHz}$ und $1 \mathrm{MHz}$. Diese Bestimmung ist den anderen Möglichkeiten vorzuziehen, weil bei der reellen Komponente der Einfluß der beiden anderen Schichten in diesem Gebiet hoher Frequenzen durch kapazitive Kurzschlüsse praktisch unterdrückt ist. Am einfachsten geht man so vor, daß man denjenigen Kurvenbereich von $\mathfrak{E}_{\text {real }}$ aufsucht, bei welchem einem Frequenzintervall 3:10 das gemessene Verhältnis der reellen Komponente entspricht. In der bilogarithmischen Darstellung erscheinen Abszissen- und Ordinatenverhältnisse als Strecken $\mathrm{S}_{1}$ bzw. $\mathrm{S}_{2}$, die wir zweckmäßig an den rechtwinkligen Kanten eines Blattes Papier auftragen, wie dies in Abb. 9 angedeutet ist. Das Papier wird nun mit den Kanten parallel zum Koordinatennetz so verschoben, bis beide Marken auf der Kurve liegen. Der unter der reçten Kante 
liegende $\Omega$-Wert enthält dann die gesuchte Zeitkonstante, in unserem speziellen Falle also

$$
\tau_{3}=\frac{\Omega}{6,28 \cdot 10^{6}} \sec ,
$$

womit auch $\varkappa_{3}$ als Begrenzung der zweiten und Anfang der dritten Exponentialschicht festgelegt ist, nämlich.durch:

$$
\varkappa_{3}=\varepsilon / 4 \pi \tau_{3} .
$$

Die restlichen drei Konstanten $d_{1}, \alpha_{1,3}, \alpha_{3}$ lassen sich dann in einfacher Weise mit dem Gleichungssystem (13b) bestimmen. Da wir nur 3 Gleichungen benötigen, beschränken wir uns auf die reellen Anteile des Scheinwiderstandes, dessen Abnahme gerade die sukzessiven Kurzschlüsse der hochohmigen Schichten mit zunehmender Frequenz am deutlichsten widerspiegelt. Durch Einsetzen einer kleinen, mittleren und hohen Frequenz in Gl. (13b) und der zugehörigen, gemessenen Realteile ermittelt man die Konstanten $A, B$ und $C$ und damit

$$
d_{1}=A x_{1,2}, \quad a_{1,3}=1 / \varkappa_{1,2} B, \quad a_{3}=\frac{a_{1,2}}{a_{1,3} \varkappa_{3} C+1} .
$$

\section{Darstellung des Randschichtprofils der Leitfähigkeit}

Die Ergebnisse derartiger Schichtanalysen für verschiedene Gleichspannungsbelastung des

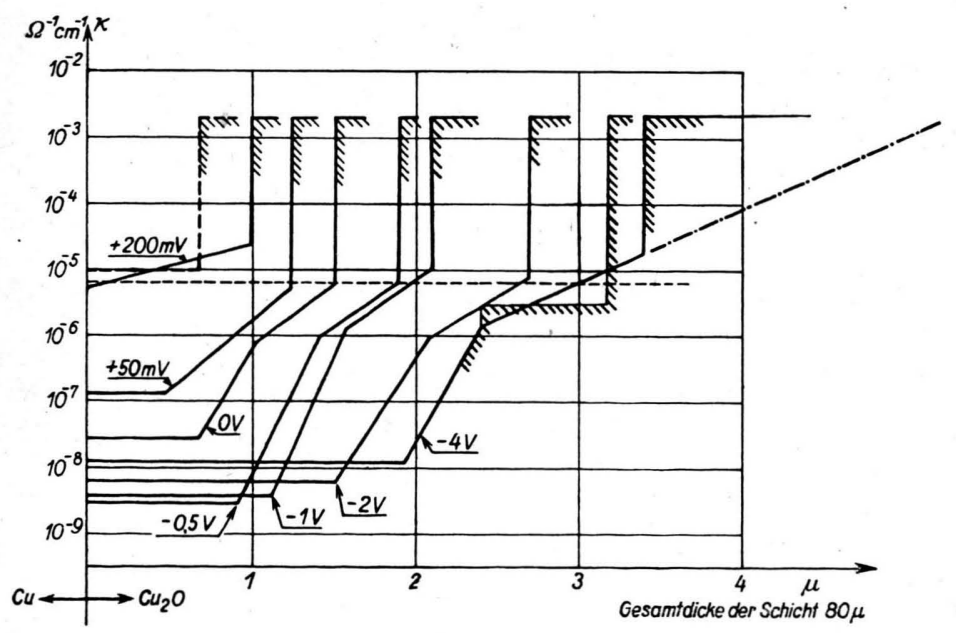

Abb. 10. Leitfähigkeitsprofile der Randschicht eines normal sperrenden Kupferoxydul-Gleichrichters bei verschiedenen Vorspannungen nach Scheinwiderstandsmessungen bei verschiedenen Frequenzen (Erläuterungen im Text).
Kupferoxydul-Gleichrichters sind in Abb. 10 dargestellt.

Der Logarithmus der Leitfähigkeit ist als Funktion des Abstandes vom Mutterkupfer aufgetragen. Als typisches Beispiel der formalen Auswertung sei zunächst das Profil bei der Vorspannung $-4 \mathrm{~V}$ besprochen. An das Mutterkupfer schließt sich die homogene Schicht mit $x_{1,2}=$ $1,15 \cdot 10^{-8} \mathrm{Ohm}^{-1} \mathrm{~cm}^{-1}$ an, die sich bis $\mathrm{zu}$ einer Entfernung von $1,95 \mu$ erstreckt. Hierauf folgt die Mittelschicht, die bis zu dem $\chi_{3}$-Wert $1,33 \cdot 10^{-6}$ $\mathrm{Ohm}^{-1} \mathrm{~cm}^{-1}$ bei etwa $2,4 \mu$ reicht, und schließlich die dritte Schicht, welche nach der formalen Auswertung zunächst bis zu beliebig hohen $x$-Werten fortgesetzt werden kann. Wir haben jedoch bereits in Abschn. III ausgeführt, daß sie als einigermaßen brauchbare Näherung höchstens bis zu Leitfähigkeiten von $6 \cdot 10^{-6} \mathrm{Ohm}^{-1}$ $\mathrm{cm}^{-1}$ angesehen werden darf (Grenze angedeutet durch die gestrichelte Linie). Ihre Fortsetzung nach größeren Schichtdicken würde überdies, wie im strichpunktierten Verlauf angedeutet, bereits bei etwa $14 \mu$ zu metallischer Leitfähigkeit führen, was natürlich völlig sinnlos ist. Anhaltspunkte für ihre Abgrenzung ergeben sich nun glücklicherweise aus dem Abbau der Randschicht mit abnehmender Sperrspannung und Übergang zur Flußrichtung hin. Bei $50 \mathrm{mV}$ ist eine Gliederung der beiden letzten Schichten schon nicht mehr möglich und auch bereits ein gewisser Spielraum für dieDicke der homogenen Schicht und die Neigung der Exponentialschicht bei der Anpassung des Frequenzganges vorhanden. Bei $200 \mathrm{mV}$ schließlich erlaubt uns die Nebenbedingung (7) nur noch eine Aufteilung der Schicht in einen frequenzabhängigen Bereich und einen konstanten Vorwiderstand (Ersatzschaltung Abb.1); oder, was damit gleichbedeutend ist, die Darstellung des Frequenzganges durch eine einzige Elementarfunktion $\mathfrak{}$. Beide Möglichkeiten sind angedeutet. Mit genügender Sicherheit kann man jedoch angeben, daß bei $200 \mathrm{mV}$ der bei $1000 \mathrm{kHz}$ frequenzabhängige Bereich höchstens bis etwa $1 \mu$ reicht. Der zugehörige Vorwiderstand ergibt sich zu $3,85 \mathrm{Ohm} / \mathrm{cm}^{2}$. Dieser unterscheidet sich praktisch nicht mehr vom Ohm- 
schen Bahnwiderstand bei hohen Flußspannungen. Als mittlere Grundleitfähigkeit des Kupferoxyduls ohne Randschicht erhält man somit bei S0 $\mu$ Dicke der Gesamtschicht.

$$
x_{0} \approx \frac{80 \mu}{3,85 \Omega \mathrm{cm}^{2}}=2 \cdot 10^{-3} \mathrm{Ohm}^{-1} \mathrm{~cm}^{-1} \text {. }
$$

Die Randschicht baut sich also auf einem Untergrund mit einer Leitfähigkeit dieser Größenordnung auf. Die Exponentialschicht muß daher spätestens bei diesem $\varkappa_{0}$-Wert abgebrochen werden. Als äußerste Grenze der Randschicht $(-4 \mathrm{~V})$ ergibt sich danach die Dicke $5 \mu$. Dieser Bereich ist aber klein gegenüber der Gesamtdicke der Oxydulschicht von $80 \mu$, so daß wir sicher keinen großen Fehler begehen, wenn wir für den Restteil der Schicht bei allen Spannungen einen frequenzunabhängigen Widerstand von $3,85 \mathrm{Ohm} /$ $\mathrm{cm}^{2}$ annehmen. Da dieser Wert aber bereits in der Exponentialschicht ebenfalls als frequenzunabhängiger Bestandteil enthalten ist, muß er von dieser abgetrennt und auf den Restteil der Schicht mit der mittleren Belegung $\varkappa_{0}$ verteilt werden. Alle Exponentialschichten sind infolgedessen bei einem bestimmten Grenzwert $\varkappa_{\mathrm{gr}}$ steil abzubrechen. $\chi_{\text {gr }}$ ist dadurch bestimmt, daß der Teilwiderstand der Exponentialschicht für $\varkappa>\varkappa_{\mathrm{gr}}$ gerade $\Delta R=3,85 \mathrm{Ohm} / \mathrm{cm}^{2}$ betragen muß. Hieraus folgt die Bestimmungsgleichung für $\varkappa_{\text {gr }}$

$$
\begin{gathered}
\frac{1}{a_{3}} \int_{\varkappa_{\mathrm{gr}}}^{\infty} \frac{a x}{x^{2}}=\frac{1}{a_{3} \varkappa_{\mathrm{gr}}}=\Delta R \\
\text { und aufgelöst } \varkappa_{\mathrm{gr}}=\frac{1}{3,85 \alpha_{3}} \mathrm{Ohm}^{-1} \mathrm{~cm}^{-1} .
\end{gathered}
$$

Eine so steil abgebrochene Exponentialfunktion unterscheidet sich nun in diesem Leitfähigkeitsbereich wegen nur noch mäßiger Erfüllung der Nebenbedingung (7) nicht mehr von einer $\mathfrak{5}$ Schicht mit etwas modifizierter mittlerer Zeitkonstante. Wenn man daher von vornherein den frequenzunabhängigen Bahnwiderstand von den gemessenen Realteilen abzieht und die Analyse von der Hochfrequenzseite her vornimmt, so kommt man auf einen halbleiterseitigen Abschluß des Profils, wie er in der durchschraffierten Begrenzung bei $-4 \mathrm{~V}$ angedeutet ist. Die Entscheidung zugunsten dẹr einen oder anderen Lösung ist im Rahmen unseres Frequenzintervalls nicht möglich und kann nur durch weitere Messungen bei noch höheren Frequenzen getroffen werden.

Zum Schluß dieses Abschnittes sei noch eine Bemerkung über den Grad der Anpassung durch maximal 3 Elementarfunktionen angefügt. Eine anschauliche Vorstellung hierüber ist der wiederholt herangezogenen $\mathrm{Abb} .7 \mathrm{~b}$ zu entnehmen, in welche ein Standardbeispiel - und zwar das am wenigsten günstige - bei $0 \mathrm{~V}$ eingetragen ist. Die gestrichelte Kurve entspricht der Anpassung an die Meßpunkte. Die Abweichungen liegen bei allen Beispielen zwischen 0 und etwa $10 \%$. Eine noch bessere Anpassung kann natürlich jederzeit durch entsprechende feinere Unterteilung der Schicht vorgenommen werden, das hieße aber die Möglichkeiten einer solchen Analyse überschätzen, wie aus der weiteren Diskussion noch hervorgeht.

\section{Diskussion des physikalischen Inhaltes der Profilkurven}

Bei der Ableitung unseres Analysenverfahrens haben wir zunächst noch einige Gesichtspunkte außer acht gelassen, die aber nunmehr in Betracht zu ziehen sind.

Wenn Schichten mit örtlich variierender Leitfähigkeit vorliegen, so müssen in ihrem Innern notwendigerweise Raumladungen vorhanden sein. welche eine Nivellierung der Leitfähigkeit durch Diffusionsausgleich der beweglichen Ladungsträger verhindern. Bei Anlegen einer Wechselspannung an die Schicht ändern sich die Feldverhältnisse periodisch und damit, wegen ihrer Verknüpfung durch die Poisson-Gleichung, auch die Raumladungsverhältnisse. Wir haben nun stillschweigend vorausgesetzt, daß die Raumladungen im Innern der Schicht auch - den Spannungswechseln entsprechend - an Ort und Stelle neu entstehen oder verschwinden können. Nur in diesem Falle dürfen wir hoffen, mit einem Ersatzschaltbild des Kettenleiters die statische Verteilung der Leitfähigkeit richtig bestimmen zu können. Diese Bedingung kann nun unter Umständen überhaupt nicht oder nur teilweise erfüllt sein. Es ist denkbar, daß die Raumladungsdichte im Innern auf einen bestimmten Wert begrenzt sein kann, oder aber die Nachlieferung aus den kontinuierlich verteilten Quellen bei schnellen Spannungswechseln zu langsam erfolgt. In diesen Fällen wird das Raum- 
ladungsdefizit im Innern an den Schichträndern aufgebaut, was nach außen hin so in Erscheinung tritt, als ob der Kettenleiter durch eine mehr oder weniger große Kapazität überbrückt ist. Trotz örtlich variierender statischer Leitfähigkeitsverteilung finden wir im ersten Fall ein Ersatzschaltbild mit Ohmschem Widerstand und Parallelkapazität, also eine quasihomogene Verteilung von $\varkappa$, während im zweiten Fall das Profil etwas nivelliert erscheint mit Leitfähigkeitssprüngen von einer Teilschicht zur anderen. Der physikalische Inhalt der Schichtprofile läßt sich daher nur im Rahmen theoretischer Grundvorstellungen ausdeuten.

Von den zahlreichen Versuchen, den Leitungsmechanismus der Systeme Metall-Halbleiter zu erklären, haben sich zuletzt die Ansätze von N. F. Mott ${ }^{5}$, besonders aber diejenigen von W. Schottky ${ }^{2}$ als fruchtbar erwiesen. Nach Mott baut sich der anomale Spannungsabfall innerhalb eines durch seine chemische Struktur festbegrenzten a priori störstellenfreien Randgebietes auf. S ch o t t ky erklärt dagegen gerade die Entstehung einer solchen, von beweglichen Ladungsträgern (Defektelektronen im Falle des Kupferoxyduls) weitgehend entblößten Schicht allein durch elektrische Wirkungen. Eine über dem Metall nach glühelektrischem Vorbild stehende Elektronenwolke prägt dem Halbleiterrand eine Verarmung an Defektelektronen ein, welche ihr Gegengewicht in einem komplementären Raumladungsfeld findet. Die Raumladungen sind ortsfest und haben ihre Quellen in negativ geladenen Störstellenresten (Akzeptoren), welche ohne die Randstörung in einem rein thermischen Dissoziationsgleichgewicht mit den Defektelektronen und den neutralen Störstellen (Donatoren $=$ Spenderstellen) zu denken sind. Die chemische Struktur der Randschicht braucht sich nicht von dem Restteil des Halbleiters zu unterscheiden. Eine chemische „Sperrschicht“ spielt mehr oder weniger die Rolle eines günstigen Untergrundes für den Aufbau der unipolar leitenden Randzone.

Nach Schottky kann sich nun vor allem die Verarmungs- und Raumladungsschicht bei Stromdurchgang örtlich ausbreiten (Sperrichtung) bzw. zusammenziehen (Flußrichtung); sie ist nicht an die Begrenzung durch eine besondere chemische Struktur der Randzone gebunden.

5 N. F. M ot t, Proc. Roy. Soc. [London], Ser. A 171, 27 [1939].
Das ist aber gerade eine sehr augenfällige Eigenschaft unserer Leitfähigkeitsprofile, welche damit nachhaltig zugunsten dieser Auffassung sprechen*. Wir werden aber zeigen, daß die spannungsabhängigen Profile außer ihrer örtlichen Ausbreitung noch feineren Zügen dieser Theorie entsprechen, welche einem so direkten Nachweis bisher nicht zugänglich waren.

Im Rahmen der Schottkyschen Theorie werden wir zunächst unsere Schichtprofile nach folgenden 3 Typenfällen zu klassifizieren suchen:

1. Fall der reinen Erschöpfungsrandschicht. Die Störstellen (Donatoren) sind bei der Versuchstemperatur vollkommen dissoziiert in Defektelektronen und ortsfeste, negativ geladene Störstellenreste (Akzeptoren). An der Metallelektrode ist durch die Einprägung der Verarmung an Defektelektronen eine Akzeptorendichte entstanden, welche der ursprünglichen Donatorendichte wegen vollkommener Dissoziation zahlenmäßig gleichzusetzen ist (Erschöpfung der Donatoren).

2. Fall der reinen Reserverandschicht. Die Donatoren sind bei der Versuchstemperatur nur teilweise dissoziiert und die Randverarmung ist nicht hoch genug, um vollständige Dissoziation in der Randzone zı erzwingen. Es ist also eine Donatorenreserve vorhanden, welche gegebenenfalls ein Defizit der Raumladungsdichte an Ort und Stelle ausgleichen kann.

3. Fall der Erschöpfungsrandschicht mit vorgelagerter Reserveschicht. Nachhaltige Störung des thermischen Dissoziationsgleichgewichtes durch eingeprägte Randverarmung und dadurch erzwungene Erschöpfung der

* Es ist jedoch ausdrücklich darauf hinzuweisen, daß der beim Kupferoxydul-Gleichrichter sicher vorhandenen ,chemischen Sperrschicht" damit keine zweitrangige Bedeutung für den technischen Gleichrichter zugewiesen werden soll. Diese ist vielmehr ein Ergebnis langjähriger technischer Entwicklung als besonders tragfähiges Fundament der hier in Erscheinung tretenden Raumladungswirkungen anzusehen und in diesem Sinne von entscheidender Bedeutung für die Güte des Gleichrichters. Hinsichtlich ihres chemischen Aufbaues (Phasengleichgewicht $\mathrm{Cu}-\mathrm{Cu}_{2} \mathrm{O}$ in der Randzone) vgl. F. W a ibel $\mathbf{4 b}$. Eine Methode zur Bestimmung der ursprünglichen chemischen Verteilung neutraler Störstellen wurde von $\mathrm{Sch}$ ottky 2c angegeben. Die Verträglichkeit des dort ermittelten Störstellenprofils mit den hier gefundenen Profilen der Wechselstromleitfähigkeit wäre allerdings ein Gegenstand weitergehender Untersuchungen. 
Donatoren mit halbleiterseitigem Übergang in ein Reservegebiet, entspr. dem Abklingen der Randverarmung.

Das Wechselstromverhalten einer Raumladungsschicht ist nun in einer Arbeit von E. $\mathrm{Spenke}{ }^{6}$ eingehend durchgerechnet worden. Wir finden dort als wesentliches Ergebnis, daß eine Erschöpfungsrandschicht sich trotz steilen Leitfähigkeitsanstieges wie eine in $\varkappa$ homogene Schicht benimmt, weil die Raumladungsdichte wegen Erschöpfung der Donatoren keiner Variation mehr fähig ist. Lediglich durch Einbeziehung weiterer halbleiterseitiger Gebiete kann die Raumladung verändert werden. Die Ladungen können nur an den Rändern pulsieren.

Auf Grund dieser Ergebnisse kann der an das Mutterkupfer angrenzende Teil unserer Leitfähigkeitsprofile als Erschöpfungsrandschicht gedeutet werden. Die Schicht ist aber dann nur quasihomogen in $\varkappa$, und $\varkappa_{1,2}$ selbst ist ein Mittelwert. Der wahre $x$ - $x$-Verlauf in dieser Schicht ist dann durch Wechselstrommessungen nicht zu erschließen.

Die zweite Schicht weist sich auch bei den Wechselstrommessungen als Gebiet örtlich variierender Leitfähigkeit aus. Ihr Verhalten entspricht somit demjenigen einer Reserveschicht. Es sind örtliche Raumladungsquellen vorhanden, die eine Feldsteuerung der Raumladungsdichte im Innern der Schicht erlauben. Die von Sch ot t k y ${ }^{2 c}$ ausgesprochene Vermutung, daß beim Kupferoxydul-Gleichrichter anschließend an die Erschöpfungsrandschicht ein Gebiet mit Reservecharakter vorhanden ist, findet damit ihre experimentelle Bestätigung. Man könnte nun versucht sein, den Reservecharakter noch zwingender herauszustellen. Eine solche Schicht muß nach der Theorie einen quadratischen Anstieg der Leitfähigkeit mit wachsender Schichtdicke aufweisen. Die Anpassung dieses Schichtabschnittes durch eine Schmiegungsparabel schiene danach das Gegebene. Nun kann man sich bei der numerischen Auswertung leicht überzeugen, daß die beiderseitig vorgelagerten Schichtanteile den Frequenzgang einer dazwischenliegenden reinen Reserveschicht so nachhaltig stören, daß ihre genaue Begrenzung nicht möglich ist. Die Anpassung durch die Exponentialfunktion ist daher sinngemäß als Kompromißlösung aufzu-

${ }^{6}$ E. S p e n ke, Wiss. Veröff. Siemens-Werke 20, 40 [1941]. fassen, welche die Randstörungen der zweiseitig begrenzten Reserveschicht mit enthält. Es sei jedoch darauf hingewiesen, daß gerade die Knickstelle, an der die Exponentialfunktionen zusammenstoßen, den $x$ - $x$-Verlauf in eine Parabel hineinzwingt. Man wird daraus schließen dürfen, daß in dem Frequenzgebiet, welches gerade diesen Schichtteil freilegt $(\omega \varepsilon / 4 \pi \varkappa=1)$ die Randstörung durch die Erschöpfungsrandschicht schon abgeklungen und diejenige der halbleiterseitigen Anschlußschicht noch nicht wirksam ist.

Wenn wir nun mit der bisherigen Interpretation theoretische Vorstellungen haben stützen können, erscheint demgegenüber der weitere Verlauf des Profils in einer halbleiterseitigen Randzone auch bei Einbeziehung des Spielraumes in der Anpassung ( $\mathfrak{h}$ - oder abgebrochene (F-Schicht) als völliges Novum. Die halbleiterseitig steile Kante steht im Widerspruch zu der Erwartung, daß die Reserveschicht allmählich auf den Untergrund (quasineutrales Gebiet) übergeht. Obwohl der Steilabfall nicht aus den Hochfrequenzmessungen selbst, sondern aus der Berücksichtigung des Bahnwiderstandes gefolgert wurde, kann sein Auftreten nur dadurch erklärt werden, daß unsere $\varkappa$-Bestimmung durch die HF-Messung entweder über die ganze Schicht oder im letzten Teil einen flacheren Verlauf ergeben hat, als der statischen Verteilung entspricht. Dann müssen wir folgerichtig den Widerstandsüberschuß, welcher in einer zu flachen Exponentialfunktion enthalten ist, durch eine Steilkante ausgleichen. Diese Abflachung des Wechselstromprofils können wir entweder dadurch erklären, daß der Ausgleich der Raumladungsdichte über das Dissoziationsgleichgewicht bei hohen Frequenzen nicht mehr rasch genug vonstatten geht, oder aber die Schicht in ihrem letzten Ausläufer die Fähigkeit wieder verliert, die Ladungspulsationen aus räumlich verteilten Quellen zu bestreiten. Die letztere Vorstellung würde dem Anschluß der $\mathfrak{S}$-Schicht mit beiderseitigen Sprungstellen der Wechselstromleitfähigkeit entsprechen.

Nach einer Abschätzung der Zeit, welche verstreicht, bis die Raumladungsdichte sich einer plötzlichen Spannungsänderung angepaßt hat, fand Schottky ${ }^{2 c}$ für den Selengleichrichter eine Abklingzeit von $\approx 1^{-10} \mathrm{sec}$, unter $\mathrm{Zu}$ grundelegung einer mittleren Beweglichkeit der Defektelektronen in Selen von $1 \mathrm{~cm} \mathrm{sec}^{-1} / \mathrm{V} \mathrm{cm}^{-1}$. 
Dieser Wert dürfte beim Kupferoxydul-Gleichrichter mit etwa 50facher Beweglichkeit der Defektelektronen (nach W.Vogt ${ }^{7}$ ) noch erheblich unterschritten sein. Da die gemessenen, äußeren Zeitkonstanten im Sperrgebiet aber noch über $10^{-8}$ sec liegen, ist die Deutung des scheinbaren $\varkappa$-Verlaufes als Auswirkung eines solchen zeitlichen Effektes nicht tragbar, es sei denn, daß die Grundlagen der Abschätzung einer inneren Zeitkonstante nicht zutreffend sind.

Die zweite Möglichkeit, daß nämlich der letzte Teil der Randschicht die Fähigkeit der Raumladungssteuerung überhaupt nicht aufweist, ist daher wahrscheinlicher.

Von Schottky selbst stammt diese letztere Auffassung, die in einer noch unveröffentlichten, umfassenden Arbeit* ..Zur Theorie der Randschichtgleichrichter (Darstellung und Fortführung nach dem Stand von 1947)“ eingehend begründet wird, und über die mit freundl. Einverständnis ihres Verf. hier noch einige Angaben folgen sollen.

Auf Grund einer von C. W a g n e r und Mitarb. ${ }^{8}$ beobachteten Anomalie in der Sauerstoffdruck-Abhängigkeit des Widerstandes von $\mathrm{Cu}_{2} \mathrm{O}$ von 700 bis $900^{\circ} \mathrm{C}$ im Gebiet kleinster Sauerstoffdrucke sowie aus einer Gesamtdiskussion der beobachteten Temperaturgänge (vgl. auch Feldmann ${ }^{9}$ ) kommt Schottky zur Postulierung eines Nebeneinanders zweier Donatorenarten (Kupferlücken und Sauerstofflücken**) mit verschiedenem Ladungscharakter und verschiedener Dissoziationsenergie. Dieser Zustand soll sich in der Randzone, in welcher der Sauerstoffmangel am größten ist, besonders stark auswirken (,Chemische Sperrschicht"). Unter dieser Voraussetzung ist im Anschluß an das Neutralgebiet eine Zone denkbar, in der die eine Donatorenart noch nicht nennenswert, die andere aber restlos dissoziiert ist, so daß im Endeffekt eine örtlich und zeitlich praktisch unveränderliche Raumladungsdichte resultiert. Dieser Schichtanteil be-

7 W. Vogt, Ann. Physik 7, 183 [1930].

* Hr. Prof. Sch ot t ky hat mir freundlicherweiso das Manuskript dieser Arbeit zugesandt, in welchem u. a. auch meine Messungen nach einem von K. L e h ov e c 1944 angegebenen differentiellen Verfahren analysiert sind.

8 C. Wagner u. Mitarb., Z. physik. Chemie, Abt. B 37, 148 [1937]. nimmt sich Wechselspannungen gegenüber wie eine Erschöpfungsrandschicht, in unserer Ausdrucksweise wie eine (quasihomogene) $\mathfrak{S}$-Schicht. Der letzte Teil des Leitfähigkeitsprofils könnte somit nach Sch ottky als Gebiet einer solchen „Pseudoerschöpfung“ aufgefaßt werden. Man wird jedoch weitere Messungen mit Ausrichtung auf diese Fragestellung abwarten müssen, ehe die zwar plausible, aber von Sch ottky selbst als heuristisch bezeichnete Vorstellung endgiiltig bestätigt werden kann.

Wir fassen die Ergebnisse zusammen: Dis Randschichtprofil der Leitfähigkeit beim $\mathrm{Cu}_{2} \mathrm{O}$ Gleichrichter dehnt sich in vollem Umfange mit zunehmender Sperrspannung aus und zieht sich in Flußrichtung wieder zusammen. Eine spainungsunabhängige örtliche Begrenzung der hochohmigen Randzone durch die besondere chemische Struktur der Randschicht ist nicht festzustellen. Damit wird ein Hauptmerkmal der Schottkyschen Raumladungstheorie durch die Hochfrequenzmessungen bestätigt. Im Rahmen dieser Theorie ist der erste Teil der Schicht als Erschöpfungsgebiet, der zweite als Reservegebiet zu deuten. Halbleiterseitig ergibt sich, entgegen der Erwartung, eine scheinbare Steilkante des Leitfähigkeitsprofils. Daraus wird geschlossen, daß sich der Reservecharakter der. zweiten Schicht allmählich verliert und die dritte Schicht im Sinne unserêr Analyse als quasihomogen und nach einer Hypothese von Schottky als Gebiet einer Pseudoerschöpfung anzusprechen ist.

Hrn. Prof. Dr. W. Schottky danke ich für die freundl. Uberlassung des Auszuges aus meinem Aktenvermerk sowie für die Zusendung seines Manuskriptes, aus welchem ich wertvolle Anregungen zur Deutung der Schichtprofile im Rahmen der Schottkyschen Theorie gewonnen habe.

Dem Leiter der Forschungsstelle für Physik der Stratosphäre, Hrn. Prof. Dr. E. R e g e n e r, bin ich dafür zu Dank verpflichtet, daß ich diese Ausarbeitung hier habe vornehmen können.

${ }^{9}$ A. F e ld m ann, Ref. F. Seitz, J. Appl. Physica 16, ว53 [1945].

** Nach Schottky ist diese zweite Störstellenart wahrscheinlich durch das Fehlen von 2 Sauerstoffionen und deren Ersatz durch 3 Elektronen gekennzeichnet; sie ist also effektiv mit einer einiach positiven Ladung behaftet. Die thermischen Gesetzmäßigkeiten lassen die Zahl der Gittermoleküle $\left(\mathrm{Cu}_{2} \mathrm{O}\right)$, die zusätzlich in der Störstelle investiert sein können, offen. 